\title{
A non destructive method for diagnostic of insulated building walls using infrared thermography in real situation
}

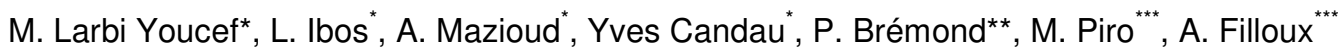

${ }^{*}$ CERTES., IUT de Créteil, Université Paris XII, 61 avenue du Général de Gaulle, 94010 Créteil, France

${ }^{* *}$ Cedip Infrared Systems, 19 boulevard Bidault, F-77183 Croissy Beaubourg, France

EDF R\&D, Site des renardières, Avenue des Renardières, Ecuelles, 77250 Moret sur Loing, France

Alpheeis, Espace Beethoven, 1200 route des lucioles 06560 Valbonne, France

\begin{abstract}
This work deals with the development of an experimental protocol for the diagnostic of multi-layered insulated building walls. The method proposed is based on active infrared thermography in step heating mode. Several commercial insulating panels composed of a plaster layer and of an insulating layer of expanded polystyrene fixed onto a building wall were investigated. A modelling of heat transfer inside the wall was performed using the thermal quadrupoles method. Results obtained show that significant differences are observed between panels of different polystyrene thicknesses. Thus, it seems possible to characterize the thermal insulation level of insulated walls using this method.
\end{abstract}

\section{Introduction}

In order to provide a method to control conformity of insulation of buildings after restoration, Protomeres project was launched under Prebat 2005 program. This work deals with the development of an experimental protocol for the diagnostic of multi-layered insulated building walls. In our previous work, a test bench was set up in order to measure front and back sides temperatures of standard panels compounded of $1 \mathrm{~cm}$ of plaster and various thicknesses of polystyrene [1]. The panels considered have insulation thicknesses of 2, 6 and $10 \mathrm{~cm}$. In the present work, the panels are fixed on walls in laboratory to test real situations. The objective of this study is to explore the possibility to characterize the thermal resistance of an insulated wall using active infrared thermography.

\section{Experimental Set up}

In this work, we investigated standard insulating panels commonly used for the thermal insulation of buildings in France. These panels are composed of two layers: a plaster layer of thickness $1 \mathrm{~cm}$ and a second layer of expanded polystyrene. Several panels with polystyrene layer thicknesses ranging from $2 \mathrm{~cm}$ to $10 \mathrm{~cm}$ were used. These panels were fixed onto a building wall. A $1 \mathrm{~cm}$-thick air layer was imposed between the rear face of the insulating panel and the wall surface. The front side of the insulating panel was coated using a black paint of 0.97 emissivity. The front side of the panels was heated using two halogen lamps of $500 \mathrm{~W}$. The selected active thermography method was the step heating. It consisted in heating the sample for a given time length (between 3600 and 9000s) and then in proceeding to observations during the whole relaxation process. Different heating power values were used. A CEDIPTM Jade long wave infrared camera was used to acquire infrared thermograms during the thermal excitation (heating phase) and after switching off the lamps (cooling phase). A cubic reflector was used to obtain a roughly constant flux density over the heated zone of the panel surface. Additional temperature measurements such as front and rear side temperature of the insulating panel, air temperature inside the reflector and room air temperature were performed using K-type thermocouples. A schematic view of the experimental setup is presented in figure 1.

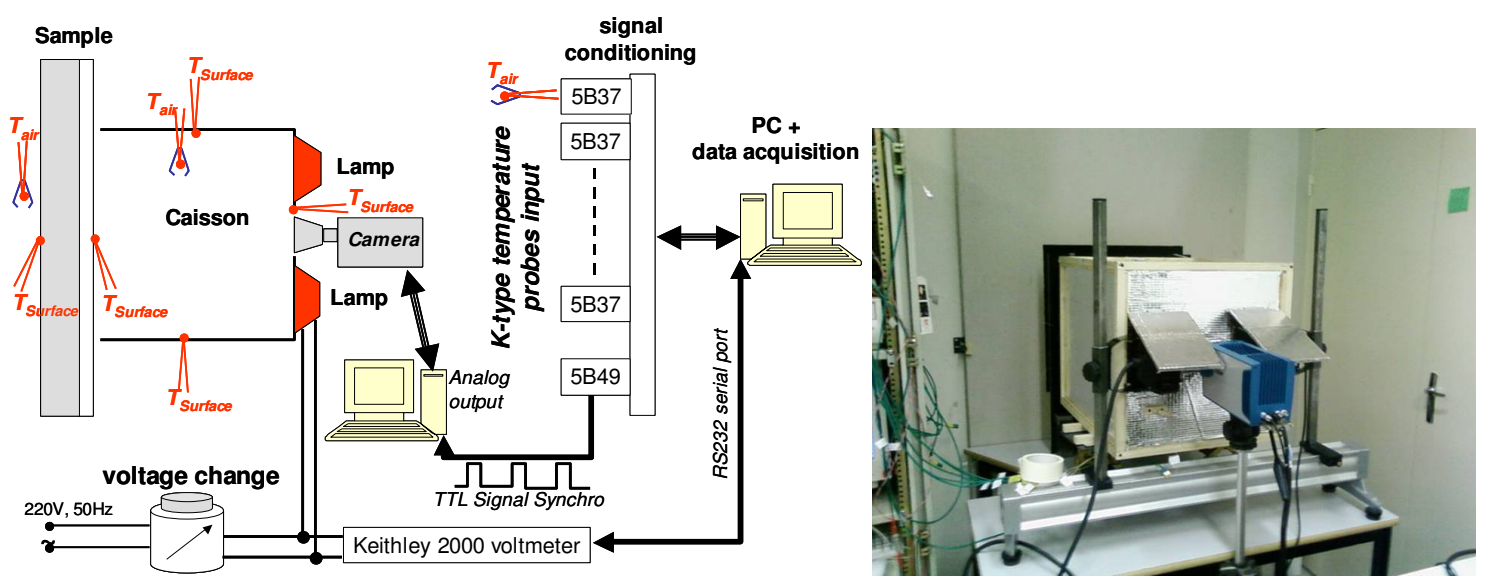

Fig. 1. Experimental set-up 


\section{Heat transfer model}

The heat transfer inside the heating panel was modeled using the thermal quadrupoles method [2]. Each layer was represented by a thermal quadrupole and the heat transfer was considered to be $1 \mathrm{D}$. This assumption was supported by numerical simulations performed under Fluent ${ }^{\mathrm{TM}}$ environment [1]. The Stefhest method was used to transform data from frequency to time domain [3]. Then, parameters such as absorbed power, heat exchange coefficient on the front face panel and thickness of the polystyrene insulating layer, were estimated by a least-square minimization method (Levenberg-Marquadt algorithm) [4].

\section{Results}

Figure 2 presents two infrared thermograms obtained on the front side of the insulating panel during the heating phase. Different regions were analyzed in order to check the uniformity of the heating power density. Figure 3 presents the variation of the panel front side temperature for three thicknesses of the polystyrene layer $(20,60$ and $100 \mathrm{~mm})$. A static regime is obtained after about 5000s. Moreover, significant differences in the evolution of the surface temperature are obtained after 1 hour of heating. In the static regime, temperature differences of a few degrees are observed between the different insulating panels investigated. Thus, it seems possible to characterize the thermal insulation level of insulated walls using this method. Besides, the measurement time is compatible with in-situ investigations. More detailed results, especially concerning identified parameters, will be presented in the full paper.

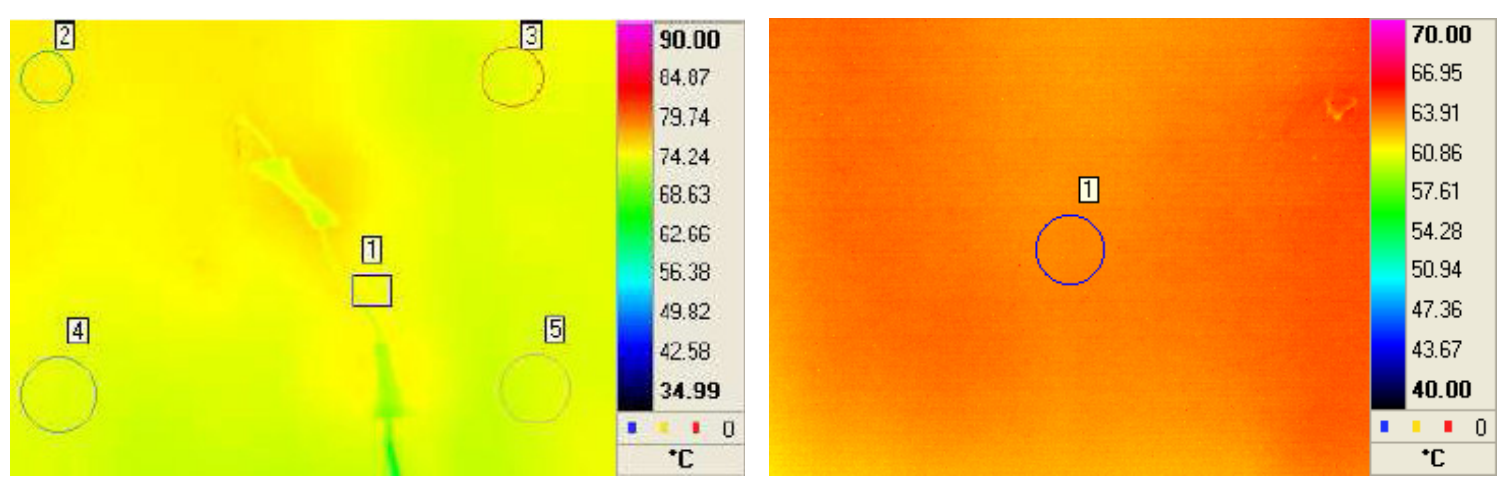

Fig. 2. Example of infrared thermograms

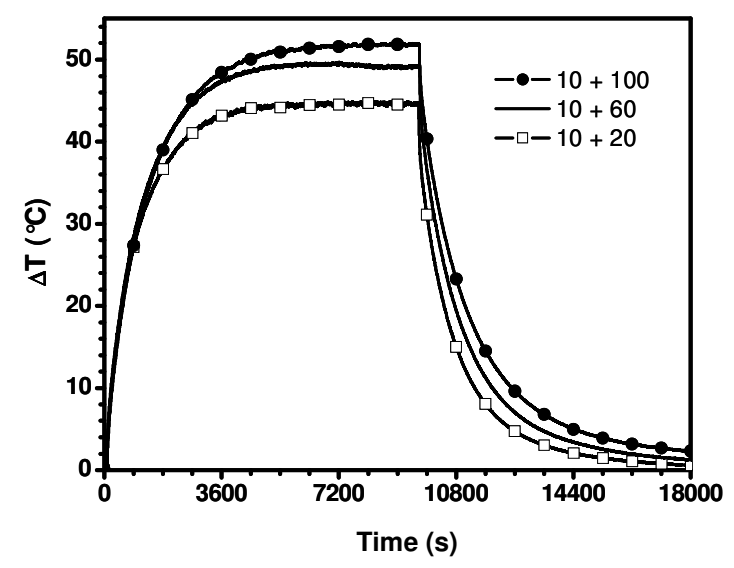

Fig. 3. Temperature variation of the front side of panels with different polystyrene insulating layer thicknesses: $20 \mathrm{~mm}(10+20), 60 \mathrm{~mm}(10+60)$ and $100 \mathrm{~mm}(10+100)$

\section{REFERENCES}

[1] M. Larbi Youcef, A. Mazioud, L. Ibos, Y. Candau, P. Brémond, M. Piro, A. Filloux, A non destructive method for diagnostic of insulated building walls using infrared thermography, Thermosense XXIX 9-13 april 2007, Orlando.

[2] H. Wang, A. Degiovanni, and C. Moyne, Int. J. Therm. Sci. 41 (2002) 125.

[3] H. Stehfest, Algorithm 368 : Numerical inversion of Laplace transform, Communication of the ACM, vol 13, $n \circ 1,(1970)$.

[4] D. W. Marquardt, An algorithm for the least squares estimation of non linear parameters, SIAM Journal, 1, 431-441, (1963). 